\title{
Giant Invasive Sacral Schwannoma Showing Chromosomal Numerical Aberrations $[-14,+18,+22]$
}

\author{
Masahiko Kanamori $^{1,2}$, Taketoshi Yasuda ${ }^{2}$, Takeshi Hori ${ }^{2}$, Kayo Suzuki ${ }^{2}$ \\ ${ }^{1}$ Department of Human Science, University of Toyama, Toyama, Japan \\ ${ }^{2}$ Department of Orthopaedics, University of Toyama, Toyama, Japan
}

Here, we report on a rare case of a giant invasive sacral schwannoma. The patient was a 58-year-old woman who had a 6-year history of non-specific buttock pain. Histological investigation confirmed the diagnosis of cellular schwannoma. The following numerical aberration was detected using the GTG-banding method for karyotypes: $47, X X,-14,+18,+22$. Cytogenetic studies of schwannomas have indicated a complete or partial loss of chromosome 22 as the most common abnormality, but this case is cytogenetically rare because of the recurrence of trisomy 22.

Keywords: Sacrum; Neurilemmoma; Cytogenetics

\section{Introduction}

Schwannoma (or neurilemmoma) is a benign tumor that is comprised of cells with the morphology and phenotype of the Schwann cell [1]. Cytogenetic and comparative genomic hybridization studies of adult schwannomas, either classic or cellular, indicate a complete or partial loss of chromosome 22, which bears the tumor suppressor gene NF2 as the most frequent abnormality [2]. In this study, we report a case of a giant invasive sacral schwannoma with a cytogenetically rare occurrence of trisomy 22 .

\section{Case Report}

A 58-year-old woman had a 6-year history of progressive left buttock pain radiating to the limb. The pain was aggravated by walking, while relieved by lumbar flexion.
She had no history of antecedent trauma or constitutional symptoms. She had objective weakness of the bilateral extensor hallucis longs, graded as $4 / 5$ by manual muscle testing. Sensory deficit was observed in the left $\mathrm{S} 1-3$ area. Deep tendon reflexes were preserved and were normal bilaterally at the knees and ankles. The patient had no fasciculation, atrophy, or upper motor neuron signs. No cutaneous stigmata of neurofibromatosis were observed.

In the computed tomography (CT) scans, a large mass was found in the upper sacrum (Fig. 1A). Angiogram showed evidence of hypervascularity (Fig. 1B). Magnetic resonance imaging (MRI) revealed a tumorous cystic lesion $(9.5 \times 8.0 \times 4.5 \mathrm{~cm})$ occupying most of the left upper sacrum. The cystic wall showed a hypointense signal on the T1-weighted images and a moderately high signal on the T2-weighted images. Homogeneous rim enhancement of the cystic lesion was observed following adminis-

Received May 9, 2012; Revised Aug 1, 2012; Accepted Aug 4, 2012

Corresponding author: Masahiko Kanamori

Department of Orthopaedics, University of Toyama,

2630 Sugitani, Toyama 930-0194, Japan

Tel: +81-76-434-7405, Fax: +81-76-434-5186, E-mail: kanamori@med.u-toyama.ac.jp 
tration of gadolinium contrast (Fig. 2). A tru-cut bone biopsy determined a histological diagnosis of schwannoma.

Bilateral laminectomy (L4-S3) was performed using a posterior midline approach to expose the spinal canal.
The cystic tumor was found to be firmly adherent to the arachnoid and nerve roots. Histopathological examination showed an Antoni A schwannoma (hypercellular) in most areas. The tumor cells were richly cellular but with
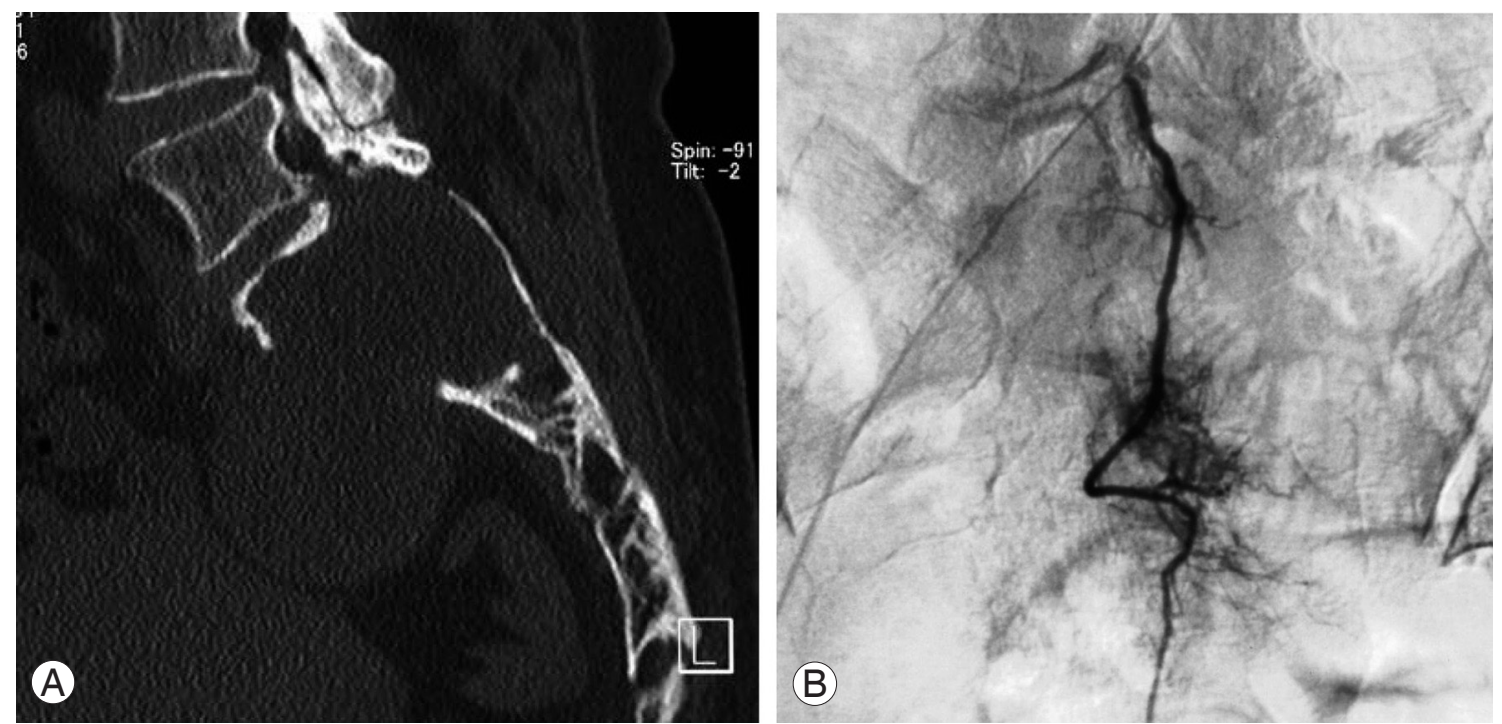

Fig. 1. (A) Coronal computed tomography of the lumbosacral spine showing destruction of the upper sacrum. (B) Angiogram showing the hypervascular tumor.
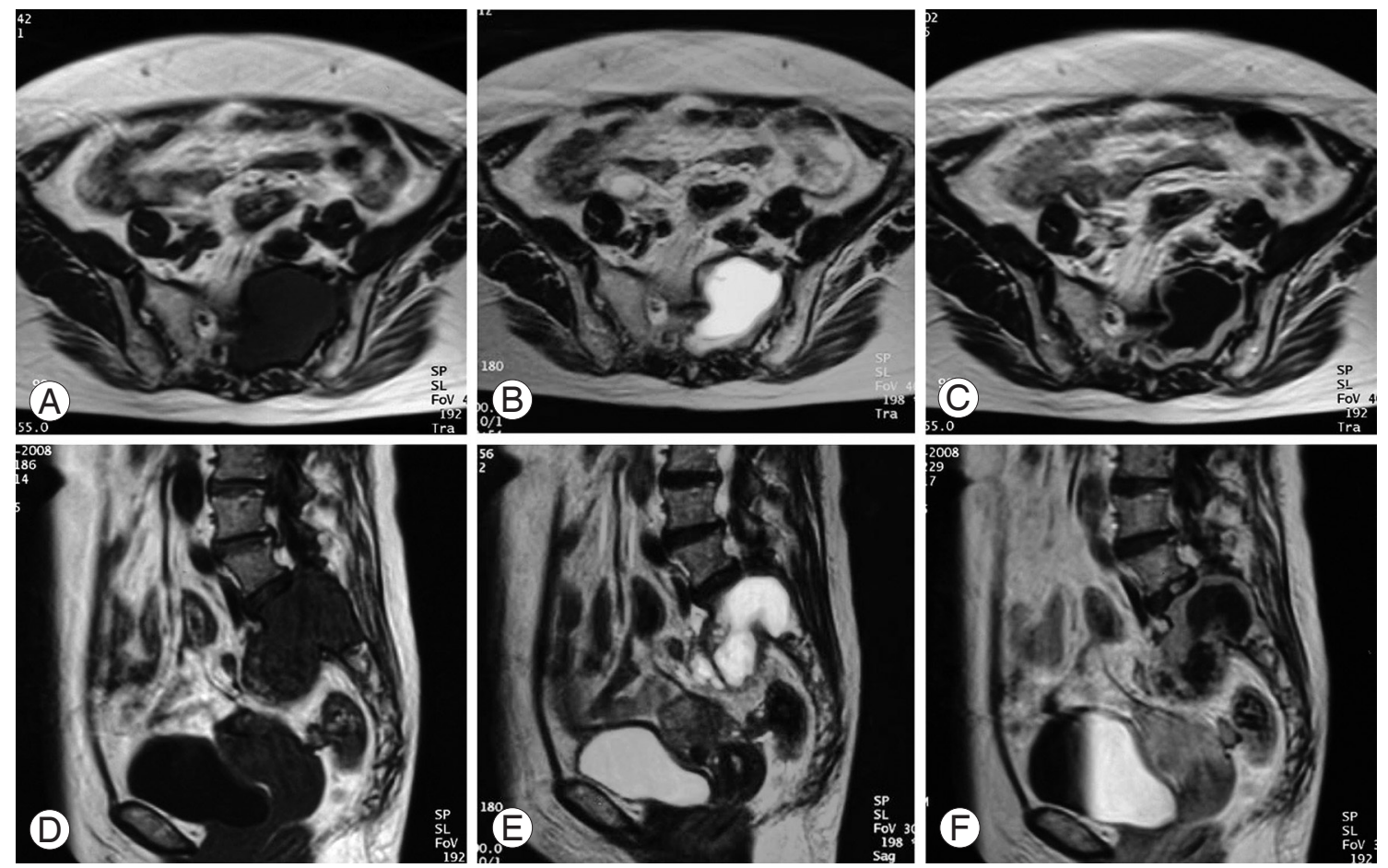

Fig. 2. Magnetic resonance imaging revealing an intraosseous tumor occupying the sacrum. Axial images showing (A) hypointensity on T1-weighted images (T1; TR: 500/TE: 12), (B) moderate hyperintensity on T2-weighted images (T2; TR: 3500/TE: 99), and (C) rim enhancement of the tumor following administration of Gd-DTPA. Sagittal images showing (D) hypointensity on T1-weighted images (T1; TR: 500/TE: 12), (E) moderate hyperintensity on T2-weighted images (T2; TR: 3500/TE: 99), and (F) rim enhancement of the tumor. 
a low mitotic count (Fig. 3A). Smaller areas showed the presence of an Antoni B schwannoma (hypocellular). No anaplasia or necrosis was detected. Immunohistochemical staining showed strong expression of the S-100 protein and glial fibrillary acidic protein (GFAP) (Fig. 3B).
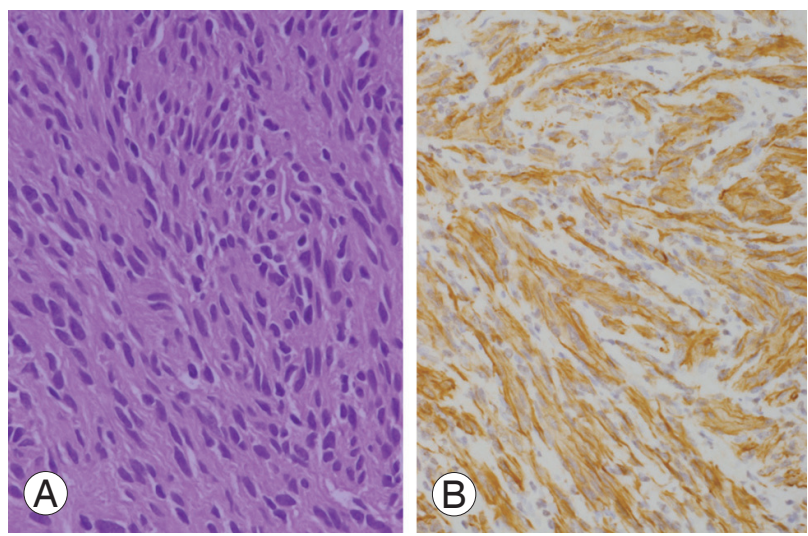

Fig. 3. (A) Schwann cells display a uniform hypercellularity with a low mitotic count $(H \& E, \times 200)$. (B) Glial fibrillary acidic protein is positive for the tumor cells (Immunohistochemical staing, $\times 200$ ).
There was no evidence of nerve infiltration or mitoses to suggest malignancy. The tumor was confirmed as a giant invasive spinal schwannoma (GISS) composed of spindle cells with elongated, hyperchromatic nuclei and abundant cytoplasm.

Cytogenetic analyses were performed on primary cultures obtained from the first surgical sample. Standard culture and harvesting procedures described previously were utilized [2]. Karyotype designation was established in accordance with the International System for Human Cytogenetic Nomenclature 2009 [3]. Twenty cells were analyzed of which 8 cells represented an abnormal clone characterized by a chromosomal numerical aberration, $47, \mathrm{XX},-14,+18,+22$ (Fig. 4). Ten cells showed various abnormal patterns, which were derived from $-14,+18$, or +22 , where the remaining 2 cells were found to be normal female cells (46,XX).

Although the tumor was incompletely excised, the patient was generally in good condition without disease progression for 3.3 years after surgery.

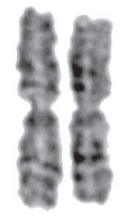

1

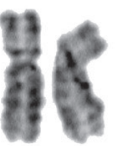

6

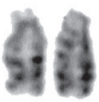

13

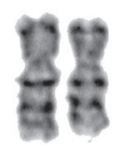

7

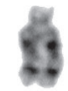

14

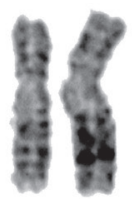

2

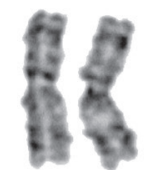

3

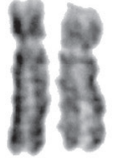

4

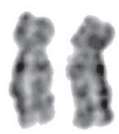

10

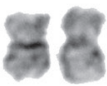

16

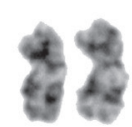

11

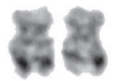

17

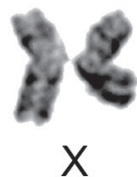

$X$

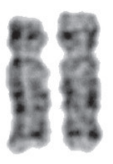

5

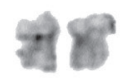

19
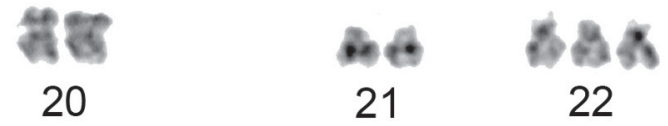

Y 


\section{Discussion}

Schwannoma is a benign tumor that arises from the embryonic neural crest cells of the nerve sheaths of peripheral or cranial nerves. In some cases, it presents as a huge mass that extends into the vertebral body and extraspinal space, as has been described in several case reports [4-7]. According to the definition provided by Sridhar et al. [4], GISS extends over more than two vertebral levels, erodes vertebral bodies, and extends posteriorly and laterally into the myofascial planes. Our case involved the sacrum, and may thus differ from typical spinal schwannomas.

Schwannoma in its classic form consists of spindleshaped cells. The histological hallmark of the schwanno$\mathrm{ma}$ is the presence of alternating areas of Antoni A (compact, hypercellular, well-organized spindle cells in a palisading pattern), palisading nuclei (Verocay bodies), and Antoni B (hypocellular, loose-textured pleomorphic cells with predominantly myxoid cytoplasm). Immunohistochemical staining showed diffuse immunoreactivity for the S-100 protein and GFAP [1]. However, many histological variants of schwannomas have been described, such as ancient schwannoma, cellular schwannoma, plexiform neurinoma, melanocytic neurinoma. Cystic changes in an ancient schwannoma is likely attributable to mucinous degeneration, ischemic necrosis, hemorrhage, and microcystic formation. However, the cellular schwannoma observed in this case study differed from the classic type in that the dense Antoni A pattern comprised $\geq 90 \%$ of the tumor area with a more uniform pattern and a lack of Verocay bodies [7]. In our case, histologically, the tumor was a cellular schwannoma with marked cystic changes. Complete surgical excision was recommended to prevent recurrence in such cases, but this was not feasible without sacrificing nerve roots over most of the sacral region.

Monosomy 22 has been reported previously, providing cytogenetic evidence in support of schwannomas. Loss of chromosomes 4, 6, 10,12,15, 17, 18, $\mathrm{X}$ and $\mathrm{Y}$ was also observed in all cases described in the literature $[2,8-10]$. On the other hand, trisomy 5, 7, 13, and 20 has also been reported. The findings of this study suggests a possible association of schwannomas with trisomy 22 , which is important because NF2 has been mapped on22q11.

In conclusion, trisomy 22 as a cytogenetic abnormality seems to be specifically associated with cellular schwannoma as well as monosomy 22 . This association needs to be confirmed by cytogenetic analysis of further cases of this tumor, which could contribute to the correct nosological classification of this entity and would provide a better understanding of its pathogenesis.

\section{Conflict of Interest}

No potential conflict of interest relevant to this article was reported.

\section{Acknowledgments}

The authors would like to thank Dr. Shinichi Hayashi, of Department of Pathology, University of Toyama, who provided pathological advices.

\section{References}

1. Enzinger FM, Weiss SW. Soft tissue tumors. 3rd ed. St. Louis: Mosby; 1995.

2. Sandberg AA, Bridge JA. Tumors of neural origin. In: Sandberg AA, Bridge JA, editors. The cytogenetics of bone and soft tissue tumors. Austin: RG Landes Company; 1994. p.229-41.

3. Shaffer LG, Slovak ML, Campbell LJ. ISCN: an international system for human cytogenetic nomenclature. Basel: Karger; 2009.

4. Sridhar K, Ramamurthi R, Vasudevan MC, Ramamurthi B. Giant invasive spinal schwannomas: definition and surgical management. J Neurosurg 2001;94:210-5.

5. Jaiswal A, Shetty AP, Rajasekaran S. Giant cystic intradural schwannoma in the lumbosacral region: a case report. J Orthop Surg (Hong Kong) 2008;16:1026.

6. Yu NH, Lee SE, Jahng TA, Chung CK. Giant invasive spinal schwannoma: its clinical features and surgical management. Neurosurgery 2012;71:58-67.

7. Woodruff JM, Godwin TA, Erlandson RA, Susin M, Martini N. Cellular schwannoma: a variety of schwannoma sometimes mistaken for a malignant tumor. Am J Surg Pathol 1981;5:733-44.

8. Couturier J, Delattre O, Kujas M, et al. Assessment of chromosome 22 anomalies in neurinomas by combined karyotype and RFLP analyses. Cancer Genet Cytogenet 1990;45:55-62.

9. Bello MJ, de Campos JM, Kusak ME, et al. Clonal 
chromosome aberrations in neurinomas. Genes Chromosomes Cancer 1993;6:206-11.

10. Joste NE, Racz MI, Montgomery KD, Haines S,
Pitcher JD. Clonal chromosome abnormalities in a plexiform cellular schwannoma. Cancer Genet Cytogenet 2004;150:73-7. 